\title{
Increased mRNA Expression of CTRP3 and CTRP9 in Adipose Tissue from Obese Women: Is it Linked to Obesity-Related Parameters and mRNA Expression of Inflammatory Cytokines?
}

\author{
Seyed Mohammad Masoodian¹, Karamollah Toolabi ${ }^{2}$, \\ Abolfazl Omidifar ${ }^{1}$, Hossein Zabihi ${ }^{3}$, \\ Ali Rahimipour ${ }^{4,5}$, Mehrnoosh Shanaki*4
}

\begin{abstract}
Background: Obesity, a medical condition with impaired adipokine secretion and function, has a detrimental effect on insulin and glucose metabolism. CTRP3 and CTRP9 are adipokines with possible roles in energy homeostasis regulation. We sought to compare CTRP3, CTRP9, and inflammatory gene expression in subcutaneous adipose tissue (SAT) and visceral adipose tissue (VAT) from obese women who underwent bariatric surgery and non-obese women as controls.

Methods: For this study, the investigators recruited 20 morbidly obese women (BMI> 35) who qualified for bariatric surgery and 20 normal-weight women $(\mathrm{BMI}<25)$ who underwent elective surgeries. Real-time PCR was performed to investigate mRNA expression of CTRP3, CTRP9, and the inflammatory genes IL1$\beta$, IL-6, MCP-1, and TNF- $\alpha$ in SAT and VAT from both obese patients and controls.

Results: We observed that CTRP3 mRNA levels were significantly greater in VAT from obese patients than from controls $(\mathrm{P}<0.0003)$. Also, patient group had higher levels of CTRP9 that control group $(\mathrm{P}<0.0026)$. Inflammatory cytokines were markedly increased in SAT of obese patients compared to controls $(\mathrm{P}<0.05)$. In addition, our results revealed a positive correlation of CTRP9 with HOMA-IR and waist circumference in VAT and CTRP3 with IL-1 $\beta$, MCP-1, and TNF- $\alpha$ in SAT.

Conclusions: Both CTRP3 and CTRP9 expression were significantly higher in VAT from obese patients than from controls, and CTRP3 expression positively correlated with inflammatory parameters. Our findings indicate that CTRP3 and CTRP9 might be important in regulating glucose metabolism and obesity-related conditions such as inflammation.
\end{abstract}

Keywords: Adipokine, CTRP3, CTRP9, Obesity, Obese women.

\section{Introduction}

Obesity is an important metabolic complication that has significantly increased over the past decade. According to a 2014 report, approximately 650 million adults over 18 years suffer from obesity worldwide (1). Obesity is a systemic condition that encompasses several complex metabolic pathways and inter-tissue crosstalk, and has a detrimental effect on energy

1: Department of Medical Laboratory Sciences, School of Allied Medical Sciences, Student Research Committee, Shahid Beheshti Univensity of Medical Sciences, Tehran, Iran.

2: Department Surgery, Imam Khomeini Hospital, Tehran University of Medical Sciences, Tehran, Iran.

3: Sina Hospital, Department of Surgery, school of medicine, Tehran University of Medical Sciences, Tehran, Iran.

4: Department of Clinical Biochemistry, School of Medicine, Shahid Beheshti University of Medical Sciences, Tehran, Iran.

5: Department of Medical Laboratory Sciences, School of Allied Medical Sciences, Shahid Beheshti University of Medical Sciences, Tehran, Iran.

*Corresponding author: Mehrnoosh Shanaki; Tel: +9821 22721151; E-mail: shanaki_m@sbmu.ac.ir.

Received: 26 Dec 2019; Accepted: 4 Jan, 2020 
homeostasis (2). Furthermore, as obesity progresses, the risks for cardiovascular diseases, heart attack, type 2 diabetes (T2DM), and nonalcoholic fatty liver disease (NAFLD) increase. Thus, identifying and understanding the molecules and related factors in the initiation and development of obesity, and its underlying mechanisms, are important challenges (3). Adipose tissue is an endocrine organ responsible for storing lipid droplets and releasing them as triglyceride when needed. Principally, adipose tissue has two distinct types of fat deposition; subcutaneous and visceral (4). Visceral adipose tissue (VAT) is more metabolically active than subcutaneous adipose tissue (SAT) and plays a crucial role in endocrine, metabolic, and immunological functions (4). Furthermore, adipose tissue, through secreting a wide range of biomolecules called adipokines, plays a key role in homeostasis regulation. Accumulating evidence indicates that alterations in adipokine production and function could be important in various conditions including inflammation, insulin resistance, T2DM, obesity, hypertension, dyslipidemia, and atherosclerosis (5). The $\mathrm{C} 1 \mathrm{q} /$ tumor necrosis factor-related proteins (CTRPs) are a 15-member family of adipokines that were recently discovered in mice and humans. These secretory proteins are adiponectin paralogues that are structurally similar to TNF- $\alpha$ and, due to their $\mathrm{C} 1 \mathrm{q}$ globular domains, have high homology with complement system proteins. CTRPs are widely expressed in various tissues, especially in mouse and human adipose tissue and plasma (6). Recent findings identified adipokines' roles in glucose and lipid metabolism, insulin resistance, and inflammatory pathways, bridging the gap between metabolic and inflammatory pathways (7).

Among CTRPs, CTRP3 and CTRP9 are capable of adipose-selective expression, and in vitro studies confirm their role in glucose and lipid metabolism along with whole-body metabolism regulation (6). CTRP3, also known as cartonectin and cartducin, is the most homologous (38\% in humans) of the CTRPs to adiponectin. It is released from mesenteric adipose tissue and mature adipocytes and can be found as protein in various organs, including liver, kidney, fat tissue, muscle, and spleen (8). CTRP9 is a close paralogue to adiponectin and is stored predominantly in stroma vascular cells (SVC) and adipocytes (9). As highly conserved secretory proteins, CTRP3 and CTRP9 circulate in the blood, indicating their role in endocrine metabolism and mechanisms (6). In vitro studies suggest CTRP3 as a vital part of glucose and lipid metabolism and show its involvement in suppression of inflammatory responses, increase of adipokine secretion, and spurring of cell division and differentiation (10-14). One study demonstrated a decrease in CTRP3 in dietinduced obese mice was concurrent to an increase in TNF- $\alpha$ and IL-6 levels (15). Moreover, CTRP3 serum concentration is significantly greater in T2DM and prediabetic than in healthy individuals (6). In the obesity field, the results are not conclusive; it has been reported a decline in serum CTRP3 levels in diet-induced obese (DIO) mice while it was observed that CTRP3 level significantly increased in leptin-deficient ob/ob mice ob/ob mice (10). To date, few studies have attempted to compare CTRP3 expression in fat tissue from obese and non-obese individuals. Further research is needed to address these issues.

Recently, CTRP9 has attracted attention from researchers. Studies show CTRP9 expression is generally greater in men than women and involved in lipid, glucose, and inflammatory processes (16). One study reported that CTRP9 expression was high in DIO and T2DM mice; but in transgenic mice, increased CTRP9 levels impeded DIO and T2DM (14). In addition, CTRP9 inhibition reduced insulin resistance in peripheral tissues and increased plasma insulin, consequently mediating insulin signaling pathways impairment and reducing insulin tolerance (16). Also, CTRP9 expression in fat tissue of DIO and 8-week ob/ob mice was significantly greater than in lean mice (17). CTRP9 expression is inhibited by TNF- $\alpha$, and CTRP9 can suppress pro-inflammatory genes, thus indicating a relationship between CTRP9 and pro-inflammatory factors (18).

To our knowledge, no data are available regarding CTRP3 and CTRP9 expression alterations in human adipose tissue and their possible association with metabolic indices in the 
context of obesity. Hence, we conducted the current study to investigate CTRP3 and CTRP9 expression and also and also the inflammation-associated molecules IL-1 $\beta$, IL-6, MCP-1, and TNF- $\alpha$ in SAT and VAT from obese and normal-weight. We also aimed to assess the possibility of an association between gene expression and clinical and biochemical parameters in obese patients.

\section{Materials and methods}

\section{Study design}

This case-control study was performed from July 2017 to August 2018. The study received approval from the Ethics Committee of the Shahid Beheshti University of Medical Sciences (IR.SBMU.RETECH.REC.1396.59), and all participants fulfilled written informed consent. The obese group consisted of 20 morbidly obese, nondiabetic women (BMI> 35, age 18-49) who were admitted to the general surgery department of Erfan Hospital, and by the specialist's advice qualified for Roux-en Y gastric bypass or vertical sleeve gastrectomy bariatric surgery. The control group consisted of 20 women (BMI< 25, age 1849) who were not diabetic or obese and were considered for other elective operations including cholecystectomy, abdominal hernia, gastric bypass, and inguinal, in the Sina and Loqman Hakim Hospitals' women's wards. Exclusion criteria were malignancy, autoimmune diseases, genetic disorders, mental disability, NAFLD, cardiovascular problems, acute and chronic infections, and thyroid issues. In addition, pregnant women, those who had surgery over the past six months, and/or were taking weight loss medications, were excluded from the study.

\section{Anthropometric and demographic assessment}

Demographic information was collected from the patients' medical records. Anthropometric measurements were completed before the surgeries. Height and weight were measured by tape measure and digital scale, respectively, and BMI was calculated by dividing the weight in kilograms by the square of the height in meters. Waist circumference (WC) was measured by passing the tape measure through the midpoint between the superior iliac crest and the lowest rib. Hip circumference was measured at the maximum protrusion. The waist-hip ratio was calculated from the waist and hip circumference data. Systolic and diastolic blood pressures were measured three times after the patient was calmly seated for 10 minutes and the average was recorded.

To evaluate biochemical parameters, blood samples were collected after a 12-hour fast and the samples promptly sent to Massoud laboratory (Tehran, Iran). Serum was isolated by centrifuging blood samples at $448 \mathrm{RCF}$ for $10 \mathrm{~min}$. A cobas 6000 autoanalyzer was utilized to determine alanine aminotransferase (ALT), aspartate aminotransferase (AST), alkaline phosphatase (ALP), serum creatinine $(\mathrm{Cr})$, uric acid, total cholesterol (TC), triglycerides (TG), high-density lipoproteincholesterol (HDL-C), low-density lipoproteincholesterol (LDL-C), and fasting blood sugar (FBS) levels. The hemoglobin A1c (HbA1c) as percentage was measured on a SEBIA capillary electrophoresis (CE) instrument (FRANCE), fasting insulin was assessed by electrochemiluminescence (ECL) using a cobas 6000 autoanalyzer, and high-sensitivity Creactive protein (hs-CRP) level was determined by the immunoturbidimetric method in a Roche Integra device. In addition, HOMA-IR, as the index of insulin resistance, was calculated based on this formula:

HOMA-IR $=$ FBG $(\mathrm{mmol} / \mathrm{l}) \times$ fasting insulin $(\mu \mathrm{I}$ $\mathrm{U} / \mathrm{ml}) / 22.5$

\section{Tissue sampling}

Visceral and subcutaneous adipose tissues were retrieved from the surgeon during each operation. In brief, 2-3 grams of visceral fat derived from omentum was eviscerated by the surgeon. A small aperture was cut under the skin and approximately 0.5 gm of subcutaneous fat was collected with a scalpel. Upon collection, fat tissue samples were placed in sterile Falcon tubes containing RPMI 1640 on dry ice and immediately transferred to the laboratory. The samples were rinsed thoroughly with PBS, cut into smaller pieces, placed in sterile tubes, and stored at $-80{ }^{\circ} \mathrm{C}$ for later analysis. All steps were performed sterilely.

\section{RNA extraction and real-time PCR}

To extract total RNA, the samples were first defrosted at ambient temperature. Next, 70-100 $\mathrm{mg}$ of VAT/SAT were placed in a mortar, frozen 
with liquid nitrogen, and crushed gently to a uniform powder. TRIzol solution was then added to make the sample homogenous. Total RNA was extracted with the RNeasy lipid tissue kit (cat no. 74804, QIAGEN, Germany). The extracted RNA was analyzed for quantity and quality with a NanoDrop spectrophotometer (NanoDrop $^{\mathrm{TM}}$ 2000, Thermo Fisher Scientific). Then, $1 \mu \mathrm{g}$ of each sample was reversetranscribed using the PrimeScript ${ }^{\mathrm{TM}}$ RT Reagent Kit (cat. RR037A, TAKARA BIO INC, Japan).
The primer sequences are listed in Table 1. To evaluate mRNA expression differences between the obese and control groups, real-time PCR was performed using a SYBR® Premix Ex Taq TM II kit (Cat. \#RR820L, Takara Bio Inc., Japan) on a Rotor-Gene Q instrument (QIAGEN, Germany). The data were normalized using glyceraldehyde-3-phosphate dehydrogenase (GAPDH) as the reference gene. Gene expression results were analyzed by the $2^{-}$ $\Delta \Delta \mathrm{ct}$ method.

Table 1. Primers used for real-time PCR. Listed are sense and antisense primer sequences with $5^{\prime}-3^{\prime}$ orientation.

\begin{tabular}{lll}
\hline Target gene & Sense primer & Antisense primer \\
\hline$C T R P 3$ & GAGTCTCCACAAACCGGAGG & TCACCTTTGTCGCCCTTCTC \\
$C T R P 9$ & TAGGGTCCAGGTGATGTTTCC & CCACCAGATCCTCATGGTTCAG \\
$I L-1 \beta$ & AAACAGATGAAGTGCTCCTTCC & AAGATGAAGGGAAAGAAGGTGC \\
$I L-6$ & CCAGCTATGAACTCCTTCTC & GCTTGTTCCTCACATCTCTC \\
$M C P-1$ & TCAGCCAGATGCAATCAA-TG & ATGGTCTTGAAGATCACAGC \\
$T N F-\alpha$ & GAAAGCATGATCCGGGACGTG & GATGGCAGAGAGGAGGTTGAC \\
$G A P D H$ & TGGAAGGACTCATGACCACA & AGGGGTCTACATGGCAACTG \\
\hline
\end{tabular}

Abbreviations: CTRP3: C1q/TNF-related protein-3; CTRP9: C1q/TNF-related protein-9; GAPDH: Glyceraldehyde 3-phosphate dehydrogenase; IL-1 $\beta$ : Interleukin 1-beta; IL-6: Interleukin-6; MCP-1: Monocyte chemoattractant protein 1; TNF- $\alpha$ : Tumor necrosis factor-alpha.

\section{Statistical analysis}

All statistics were analyzed using SPSS (version 16.0; SPSS Inc., Chicago, IL, USA). Continuous variables were tested for normality by the Shapiro-Wilk test. Variables with normal distribution are expressed as means \pm standard error of the means (SEM). Variables without normal distribution are displayed as median and interquartile ranges (IQR). Between-group comparisons were analyzed by the student's ttest and Mann-Whitney U test when indicated, based on normality assumption. Spearman's rho correlation coefficients were computed for the associations of genes together and for their associations with clinical variables. Multivariate analysis of covariance (ANCOVA) was performed for the effects of biochemical factors adjustment. We used the comparative CT method for mRNA expression analysis (19). For all analyses, a p-value less than 0.05 was considered statistically significant.

\section{Results}

\section{Subjects' baseline characteristics}

The subjects' anthropometric and biochemical parameters are shown in Table 2. The average
BMIs of the obese and non-obese groups were 41 $\mathrm{kg} / \mathrm{m}^{2}$ (range $36.3-45.2 \mathrm{~kg} / \mathrm{m}^{2}$ ) and $24.93 \mathrm{~kg} / \mathrm{m}^{2}$ (range $24.9-25.94 \mathrm{~kg} / \mathrm{m}^{2}$, respectively). Waist circumference, BMI, and hip circumference were significantly greater in the obese than in the control group (Table 2). Furthermore, LDL-C, FINS, HOMA-IR, TC, urea, $\mathrm{Cr}$, and hs-CRP values were significantly greater in the obese than in the control group. No significant difference was found between the two groups for SBP, DBP, WHR, HDL-C, VLDL-C, ALT, AST, ALP, or TG levels.

\section{mRNA expression levels in VAT and SAT}

CTRP3 and CTRP9 expression in VAT were significantly greater in the obese than in the control group (Fig. 1a and 1c). CTRP3 and CTRP9 expression in SAT from the two groups were not significantly different (Fig. 1b and 1d). In addition, the obese group had significantly greater expression than the controls for IL-1 $\beta$, MCP-1, IL6 , and TNF- $\alpha$ in VAT (Figs. 2a, 2c, 3a, and 3c). MCP-1 and IL-6 expression were significantly greater in SAT from the obese group than from controls (Figs. $2 \mathrm{~d}$ and $3 \mathrm{~b}$ ). IL-1 $\beta$ and TNF- $\alpha$ expression in SAT were not statistically different 
between the two groups (Figs. $2 \mathrm{~b}$ and $3 \mathrm{~d}$ ). Results of Spearman correlation analysis in whole study participants to examine CTRP3 and CTRP9 association with metabolic variables and inflammation-related genes are shown in Table 3. Here, a positive correlation exists between SAT CTRP3 expression and IL-1 $\beta$, MCP-1, and TNF$\alpha$ expression. Moreover, a positive correlation was observed between VAT CTRP3 gene expression and HOMA-IR and WC. We also found a positive correlation between VAT CTRP9 expression and WC.

ANCOVA showed CTRP3 and CTRP9 expression levels in VAT are independent of age and HOMA-IR (age/HOMA-IR-corrected $\mathrm{p}$ value< 0.05 ).

Table 2. Study subjects' clinical and laboratory characteristics.

\begin{tabular}{|c|c|c|c|}
\hline & Normal- weight group & Obese group & p value \\
\hline Age (years) & $36.5(31.5-51)$ & $35(30.5-38)$ & 0.115 \\
\hline $\mathrm{BMI}\left(\mathrm{kg} / \mathrm{m}^{2}\right)$ & $24.93(24.21-25.9)$ & $41(36.2-45.5)$ & $<0.001 *$ \\
\hline $\mathrm{WC}(\mathrm{cm})$ & $88(85-101)$ & $114(110-125)$ & $<0.001 *$ \\
\hline Hip circumference $(\mathrm{cm})$ & $101.5(92.5-107.5)$ & $130.5(120-141)$ & $<0.001 *$ \\
\hline WHR & $0.887(0.870-0.934)$ & $0.913(0.842-0.948)$ & 0.494 \\
\hline $\mathrm{SBP}(\mathrm{mm} \mathrm{Hg})$ & $120(110-120)$ & $120(110-125)$ & 0.379 \\
\hline $\mathrm{DBP}(\mathrm{mm} \mathrm{Hg})$ & $75(70-80)$ & $71.5(60-80)$ & 0.320 \\
\hline $\mathrm{FBS}(\mathrm{mg} / \mathrm{dL})$ & $91(81.25-98.7)$ & 85.05 (80.65-92) & 0.223 \\
\hline FINS & $7.615(5.41-9.86)$ & $19.45(15.8-25.32)$ & $<0.001 *$ \\
\hline HOMA-IR & $1.71(1.05-2.20)$ & $3.90(3.26-5.49)$ & $<0.001 *$ \\
\hline hs-CRP (mg/dL) & $1.8(1.045-2.845)$ & $4.84(2.675-9.750)$ & $0.002 *$ \\
\hline $\mathrm{LDL}-\mathrm{C}(\mathrm{mg} / \mathrm{dL})$ & $84.95(68.75-110.25)$ & $119.05(100.5-131.7)$ & $0.001 *$ \\
\hline VLDL (mg/dL) & $20(13.5-28)$ & $21(17-29)$ & 0.215 \\
\hline $\mathrm{TC}(\mathrm{mg} / \mathrm{dL})$ & $137.1(115.45-167.65)$ & $186.95(165.25-201.45)$ & $0.001 *$ \\
\hline $\mathrm{TG}(\mathrm{mg} / \mathrm{dL})$ & $91.55(63.15-125.5)$ & $124.9(109.95-157.65)$ & 0.061 \\
\hline $\operatorname{Urea}(\mathrm{mg} / \mathrm{dL})$ & $20.85(16.65 .27 .65)$ & $25.5(21.3-28.55)$ & $0.048 *$ \\
\hline $\mathrm{Cr}(\mathrm{mg} / \mathrm{dL})$ & $0.575(0.5-0.675)$ & $0.705(0.645-0.81)$ & $0.001 *$ \\
\hline $\operatorname{AST}(\mathrm{U} / \mathrm{l})$ & $15.8(10.9-21.1)$ & $20.65(15.55-23.55)$ & 0.064 \\
\hline $\operatorname{ALT}(\mathrm{U} / \mathrm{l})$ & $12.85(11.1-20.9)$ & $19.55(14.2-28.05)$ & 0.083 \\
\hline $\mathrm{ALP}(\mathrm{U} / \mathrm{I})$ & $63.75(51.1-87.25)$ & $70.85(62.4-83.45)$ & 0.207 \\
\hline
\end{tabular}

Abbreviations: ALT: Alanine aminotransferase; AST: Aspartate aminotransferase; ALP: Alkaline phosphatase; BMI: Body mass index; Cr: Creatinine; DBP: Diastolic blood pressure; FBS: Fasting blood sugar; FINS: Fasting insulin; HbAlc: Hemoglobin A1c; hs-CRP: High-sensitivity Creactive protein; HOMA-IR: Homeostatic model assessment of insulin resistance; LDL-C: Low-density lipoprotein-cholesterol; SBP: Systolic blood pressure; TC: Total cholesterol; TG: Triglycerides; VLDL: Very low density lipoprotein; WC: Waist circumference; WHR: Waist-to-hip ratio. Data are presented as median [inter-quartile range (IQR)]. *: $p<0.05$ indicates significant differences between the study groups.

Table 3. Comelation of CTRP3 and CTRP9 gene expression with anthropometric and laboratory variables and inflammatory marker expression.

\begin{tabular}{lllll}
\hline \multirow{2}{*}{ Variable } & \multicolumn{2}{l}{ CTRP3 gene expression } & \multicolumn{2}{l}{ CTRP9 gene expression } \\
\cline { 2 - 5 } & $\mathrm{S} A T$ & $\mathrm{VAT}$ & $\mathrm{SAT}$ & $\mathrm{VAT}$ \\
\hline \multirow{2}{*}{ BMI $\left(\mathrm{Kg} / \mathrm{m}^{2}\right)$} & $\mathrm{r}=0.065$ & $\mathrm{r}=0.346$ & $\mathrm{r}=0.092$ & $\mathrm{r}=0.262$ \\
& $\mathrm{p}=0.345$ & $\mathrm{p}=0.014^{*}$ & $\mathrm{p}=0.286$ & $\mathrm{p}=0.051$ \\
\hline \multirow{2}{*}{ WC $(\mathrm{cm})$} & $\mathrm{r}=0.207$ & $\mathrm{r}=0.499$ & $\mathrm{r}=0.172$ & $\mathrm{r}=0.266$ \\
& $\mathrm{p}=0.100$ & $\mathrm{p}=0.001^{*}$ & $\mathrm{p}=0.144$ & $\mathrm{p}=0.049^{*}$ \\
\hline \multirow{2}{*}{ HOMA-IR } & $\mathrm{r}=0.041$ & $\mathrm{r}=0.445$ & $\mathrm{r}=0.093$ & $\mathrm{r}=0.044$ \\
& $\mathrm{p}=0.402$ & $\mathrm{p}=0.002^{*}$ & $\mathrm{p}=0.284$ & $\mathrm{p}=0.393$ \\
\hline \multirow{2}{*}{ HDL-C } & $\mathrm{r}=0.039$ & $\mathrm{r}=0.388$ & $\mathrm{r}=0.212$ & $\mathrm{r}=0.473$ \\
& $\mathrm{p}=0.405$ & $\mathrm{p}=0.007^{*}$ & $\mathrm{p}=0.095$ & $\mathrm{p}=0.001^{*}$ \\
\hline \multirow{2}{*}{$\mathrm{IL}-1 \beta$ (SAT) } & $\mathrm{r}=0.243$ & $\mathrm{r}=0.185$ & $\mathrm{r}=0.166$ & $\mathrm{r}=0.094$ \\
& $\mathrm{p}=0.014 *$ & $\mathrm{p}=0.047^{*}$ & $\mathrm{p}=0.066$ & $\mathrm{p}=0.198$ \\
\hline \multirow{2}{*}{$\mathrm{IL}-1 \beta(\mathrm{VAT})$} & $\mathrm{r}=0.185$ & $\mathrm{r}=0.135$ & $\mathrm{r}=0.023$ & $\mathrm{r}=0.113$ \\
& $\mathrm{p}=0.047^{*}$ & $\mathrm{p}=0.111$ & $\mathrm{p}=0.417$ & $\mathrm{p}=0.153$ \\
\hline \multirow{2}{*}{ MCP-1 (SAT) } & $\mathrm{r}=0.217$ & $\mathrm{r}=0.175$ & $\mathrm{r}=0.243$ & $\mathrm{r}=0.173$ \\
& $\mathrm{p}=0.024^{*}$ & $\mathrm{p}=0.057$ & $\mathrm{p}=0.014 *$ & $\mathrm{p}=0.058$ \\
\hline \multirow{2}{*}{ TNF- $\alpha$ (SAT) } & $\mathrm{r}=0.318$ & $\mathrm{r}=0.217$ & $\mathrm{r}=0.141$ & $\mathrm{r}=0.190$ \\
& $\mathrm{p}=0.002^{*}$ & $\mathrm{p}=0.024^{*}$ & $\mathrm{p}=0.100$ & $\mathrm{p}=0.042^{*}$ \\
\hline
\end{tabular}

Abbreviations: BMI, body mass index; HDL-C, high-density lipoprotein-cholesterol; HOMA-IR, Homeostasis Model Assessment for insulin resistance; Il-1 $\beta$, interleukin 1 beta; MCP-1, Monocyte chemoattractant protein 1; TNF- $\alpha$, Tumor necrosis factor $\alpha$; WC, waist circumference. *Spearman correlation was used to calculate associations between variables. All $p$ values less than 0.05 were considered significant. 

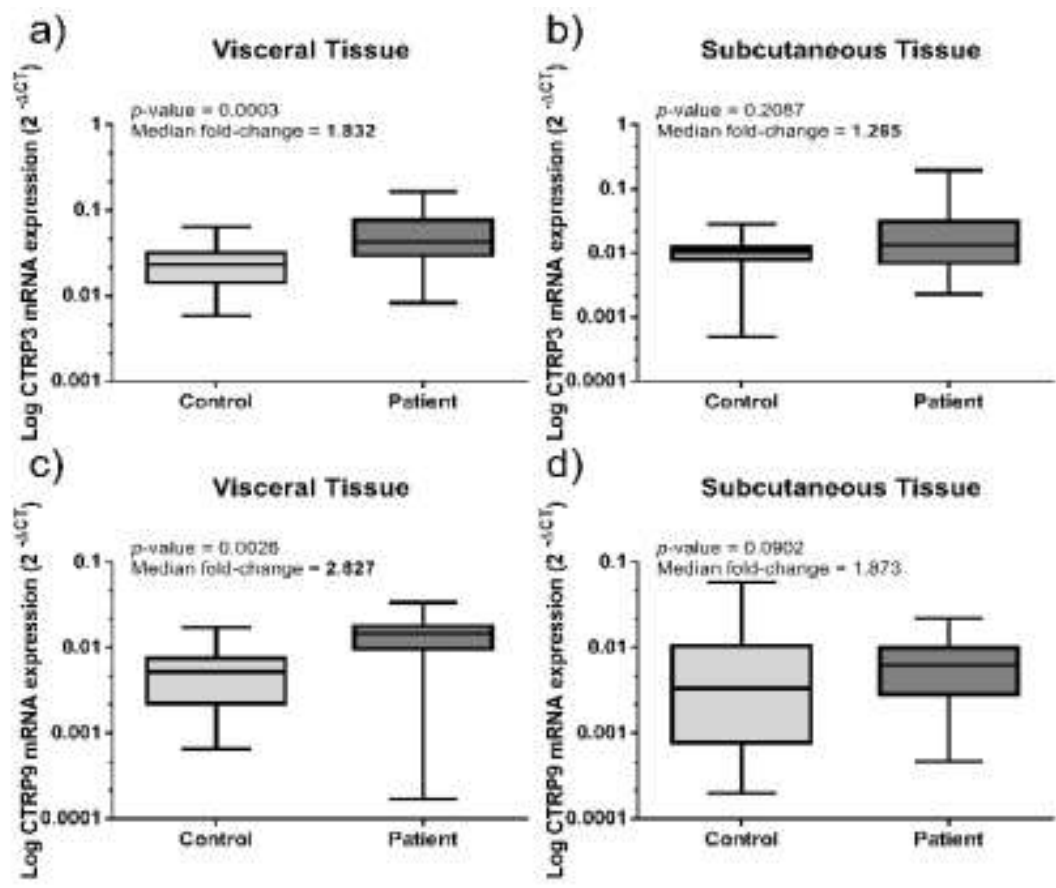

Fig. 1. Relative C1q/TNF-related protein-3 (CTRP3) and CTRP9 mRNA expression in visceral adipose tissue (VAT) and subcutaneous adipose tissue (SAT) samples from the control and patient groups. (a) CTRP3 expression was significantly greater in VAT from patients than from controls ( $\mathrm{p}=0.0003$ ); (b) No significant difference in CTRP3 expression in SAT was seen between the two groups ( $\mathrm{p}=0.2087$ ); (c) CTRP9 expression was significantly greater in VAT from patients than from controls $(\mathrm{p}=0.0026)$; (d) No significant difference in CTRP9 expression in SAT was seen between the two groups $(\mathrm{p}=0.0902)$. *The relative gene expression levels were presented as 2- $\triangle \triangle C T$ and gene expression data were transformed $\log 2$ scale to approximate a normal distribution. ${ }^{* *} \mathrm{p}<0.05$ indicates significant differences.
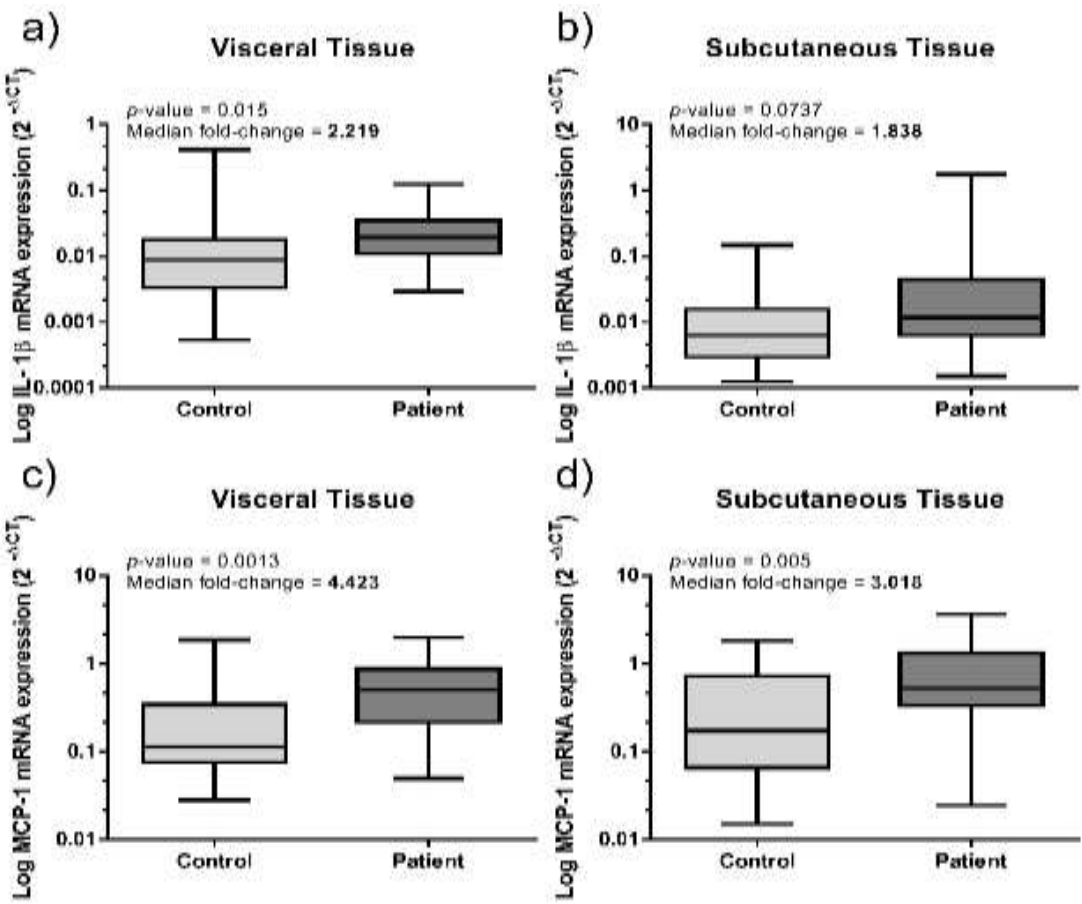

Fig. 2. Relative interleukin (IL)-1 $\beta$ and monocyte chemoattractant protein 1 (MCP-1) inflammatory cytokines expression in visceral adipose tissue (VAT) and subcutaneous adipose tissue (SAT) from the control and patient groups. (a) IL-1 $\beta$ expression was significantly greater in VAT from patients than from controls $(\mathrm{p}=0.015)$; (b) No significant difference in IL-1 $\beta$ expression in SAT was seen between the two groups ( $\mathrm{p}=0.0737$ ); ( $\mathrm{c}$ and $\mathrm{d}$ ) MCP-1 expression was significantly greater in VAT and SAT from patients than from controls $(\mathrm{p}=0.0013$ and 0.005 , respectively). *The relative gene expression levels were presented as $2-\Delta \Delta C T$ and gene expression data were transformed log 2 scale to approximate a normal distribution. $* * \mathrm{p}<0.05$ indicates significant differences. 

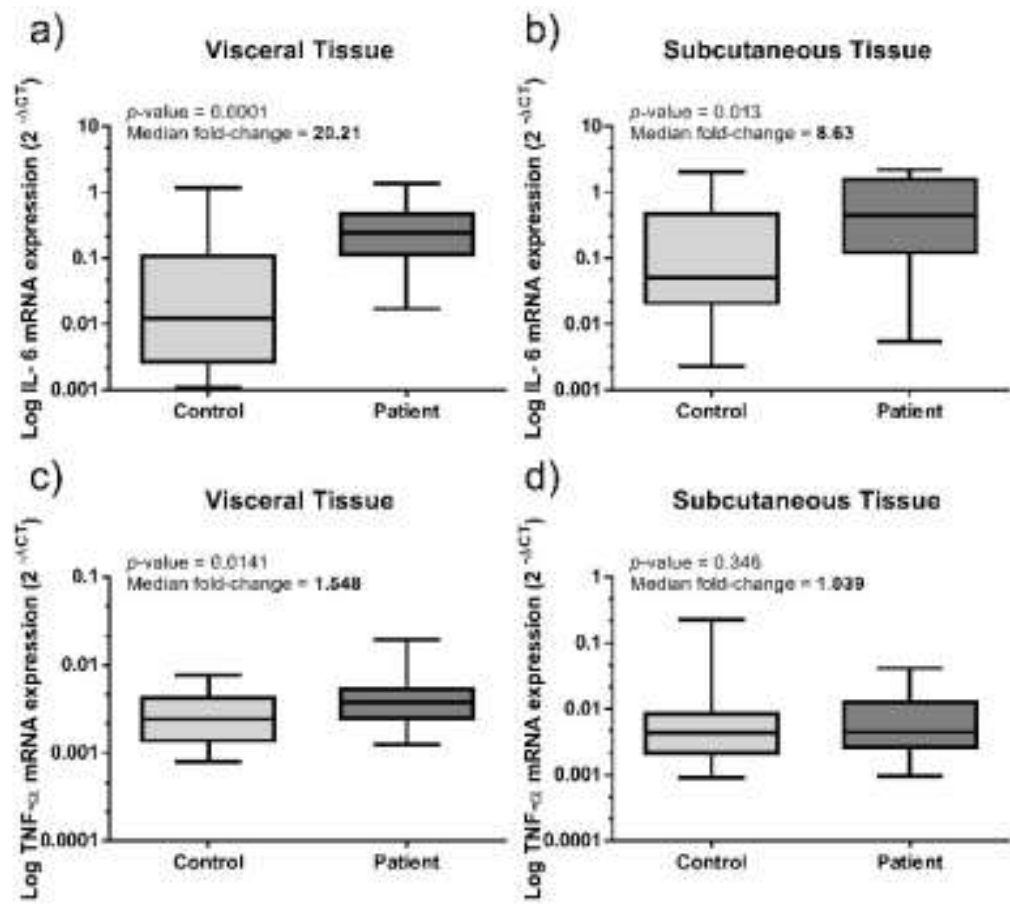

Fig. 3. Relative interleukin (IL)- 6 and tumor necrosis factor-alpha (TNF- $\alpha$ ) in visceral adipose tissue (VAT) and subcutaneous adipose tissue (SAT) samples from the control and patient groups. $(\mathrm{a}, \mathrm{b}) \mathrm{IL}-6$ expression was significantly greater in VAT and SAT from patients than from controls ( $\mathrm{p}=0.0001$ and 0.013 , respectively); (c) TNF- $\alpha$ expression was significantly greater in VAT from patients than from controls ( $\mathrm{p}=0.0141$ ); (d) No significant difference in TNF- $\alpha$ expression in SAT was seen between the two groups $(\mathrm{p}=0.346)$. *The relative gene expression levels were presented as $2-\triangle \triangle C T$ and gene expression data were transformed $\log 2$ scale to approximate a normal distribution. $* * \mathrm{p}<0.05$ indicates significant differences.

\section{Discussion}

Obesity, a condition with disrupted adipokine secretion and function, is strongly associated with T2DM, cardiovascular diseases, dyslipidemia, and hypertension $(20,21)$. Adipokines, proteins secreted from adipose tissue, have a vital role in regulating insulin signaling and energy balance $(20,22)$. CTRP3 and CTRP9 are adipokines with roles in glucose and lipid metabolism regulation and inflammation (23). Hence, to unravel underlying mechanisms for obesity we analyzed CTRP3 and CTRP9 and the inflammation-related genes IL-1 $\beta$, IL- 6 , TNF- $\alpha$, MCP-1 expression in VAT and SAT from obese and non-obese women. We also assessed the possible correlations of CTRP3 and CTRP9 levels with obesity indices and inflammatory gene expression. CTRP3 mRNA expression in VAT was significantly greater in obese women than in controls. Similar to our results, Schmid et al. observed that CTRP3 is highly expressed in SAT and VAT, and its expression might be affected by BMI and insulin (24). Moreover, Wagner et al. showed that CTRP3 serum levels were greater in obese men than in women with T2DM (20). It was recently reported that CTRP3 serum levels were greater in obese than in non-obese individuals, but no difference was found between the two groups for IL-6 or TNF (25). However, $\mathrm{Qu}$ et al. reported that patients with obesity and T2DM had lower CTRP3 serum levels than healthy controls and CTRP3 serum levels negatively correlated with IL-6. Therefore, they suggested that CTRP3 is associated with insulin resistance, possibly by regulating inflammatory pathways (26).

We also found that CTRP9 mRNA expression was significantly greater in obese women than in controls. Wong et al. showed that CTRP9 was overexpressed in obese mice and caused lowered serum glucose levels by stimulation of AMPK, Akt, and p44/42 MAPK phosphorylation in myotubes in the mice muscles (16). However, Peterson et al. revealed that obese mice had lower CTRP9 levels than lean mice due to a decrease in Akt phosphorylation and insulin-impaired signaling in mice livers (17). Moreover, Forouhi 
et al. reported similar CTRP9 serum levels in polycystic ovary syndrome (PCOS) women and healthy controls, but CTRP9 levels positively correlated with serum LDL-C and total cholesterol in these patients (27).

These discrepancies might be due to differences between animal models and humans. Animal models are often designed to exhibit certain aspects of a disease. For example, dietinduced models have been considered as polygenic models for obesity, while in others, obesity is genetically engineered (28). Notably, the point should be stressed that patient gender differences might be an important factor that should also be considered in comparing our results to others.

In our study, a significant correlation was seen between CTRP3 mRNA expression and MCP-1 and TNF- $\alpha$ in SAT, and between CTRP3 expression and BMI, WC, HOMA-IR, and HDL$\mathrm{C}$ in VAT of obese women. Also, CTRP3 expression was significantly related to IL-1 $\beta$ in VAT and SAT of obese women. Ban et al. reported that CTRP3 serum levels were lower in T2DM patients than in controls (21). In addition, no correlation was found between CTRP3 levels with glucose, hs-CRP, insulin, HOMA-IR, or leptin in their study (21). Moreover, Deng et al. showed that CTRP3 serum levels were lower in the obese group than in controls, and a significant correlation was found between CTRP3 and WC, TG, FBG, and HOMA- $\beta$ (29). They confirmed that CTRP3 can lower glucose by activating the Akt signaling pathway and suppressing enzyme expression in the liver (29). Furthermore, Wolf et al. report that CTRP3 levels were lower in the obese group than in controls. Also, serum CTRP3 levels correlated with BMI, TG, HDL, adiponectin, and female gender, which agreed with our data that showed a correlation between CTRP3 levels and BMI, HDL-C, and female gender (30). On the other hand, Zhang et al. showed that CTRP3 levels were lower in T2DM patients with NAFLD than in healthy controls, and CTRP3 levels had no correlation with BMI, HbA1c, TG, CRP, IL-6, or HOMA-IR (31). In contrast with other studies, our results showed greater CTRP3 levels in the obese woman than in controls. Obesity, a low-inflammatory condition, is associated with increased macrophage accumulation within the fat mass. The production and expression of proinflammatory molecules and chemokines have been shown to be increased in obesity (32). The anti-inflammatory role of CTRP3, through suppressing the toll-like receptor 4 (TLR4) signaling pathway (33) and inhibiting TNF- $\alpha$ and IL- 6 production in immune cells (34), has been well documented. In agreement with ample evidence for up-regulation of proinflammatory cytokines in the context of obesity in humans (35), we found greater IL-1 $\beta$, IL-6, MCP-1, and TNF- $\alpha$ expression in adipose tissues from obese patients than from controls. These results indicate that increased CTRP-3 serum levels in these women reflect a compensatory mechanism against increased inflammation in obese patients' adipose tissues.

Notably, a significant correlation was found between CTRP9 expression and WC, HDL-C, and MCP-1. In contrast to our results, Hwang et al. reported that patients with metabolic disorders had reduced serum CTRP9, and these levels inversely correlated with age, blood pressure, fasting glucose, and LDL-C (36). In addition, Wang et al. showed that CTRP9 levels were lower in coronary angiography-confirmed patients than in healthy controls, and had no correlation with BMI, HOMA-IR, TNF- $\alpha$, or IL- 6 but were positively correlated with HDL-C circulating levels (37). However, based on a recent study, CTRP9 serum levels were greater in obese woman than in controls (38). Also, there is evidence that CTRP9 levels were greater in both impaired glucose tolerance (IGT) and T2DM patients than in healthy controls, and elevated CTRP9 levels were more common in women than in men. Moreover, CTRP9 levels correlated with BMI, FBS, HbA1c, and LDL-C (39). Recently, it was shown that hypoxia causes an increase in ERK1/2 phosphorylation, but CTRP9 suppressed hypoxia-induced ERK1/2 phosphorylation and the TGF- $\beta 1 /$ ERK1/2 pathway in pulmonary arterial hypertension (PAH) patients, indicating a protective role for it in these patients (39). Therefore, the results of our study were similar to recent studies that showed greater CTRP9 levels in obese women than in controls. However, we found a positive 
correlation between CTRP9 levels and MCP-1 in obese women, providing further evidence for the possible involvement of CTRP9 in regulating inflammatory signaling pathways. In agreement this finding, Moradi et al. found a positive correlation between CTRP9 circulating levels and the inflammatory cytokines IL- 6 and TNF- $\alpha$ in patients with coronary artery disease (40). Several experimental results point to a role for CTRP9 in attenuating inflammation. For example, CTRP9 was up-regulated in macrophages and it suppressed pro-inflammatory cytokine expression in macrophage foam cells (41). Moreover, CTRP9 significantly reduced oxidized lowdensity lipoprotein (oxLDL)-induced TNF- $\alpha$ and MCP-1 expression by inhibiting nuclear factor- $\mathrm{\kappa B}$ signaling pathways in macrophages (42).

MCP-1, a proinflammatory chemokine, is produced by macrophages, human preadipocytes, and isolated mature adipocytes. A number of studies reported overexpression of this chemokine in humans and rodents with obesity (43). Therefore, it is tempting to speculate that CTRP9 gene expression is increased in a compensatory manner in adipose tissues from obese individuals to combat detrimental effects of inflammation and obesity; however, more studies are required to establish this concept.

Some limitations should be addressed; Because our study was a cross-sectional design,

\section{References}

1. Hruby A, Hu FB. The epidemiology of obesity: a big picture. Pharmacoeconomics. 2015;33(7):673-89.

2. Schwartz MW, Seeley RJ, Zeltser LM, Drewnowski A, Ravussin E, Redman LM, et al. Obesity pathogenesis: an Endocrine Society scientific statement. Endocr Rev. 2017;38(4):267-296.

3. Egger G, Dixon J. Beyond obesity and lifestyle: a review of 21st century chronic disease determinants. Biomed Res Int. 2014;2014,731685

4. Ibrahim MM. Subcutaneous and visceral adipose tissue: structural and functional differences. Obes Rev. 2010;11(1):11-8.

5. Ouchi N, Parker JL, Lugus JJ, Walsh K. Adipokines in inflammation and metabolic disease. Nat Rev Immunol. 2011;11(2):85-97. we could not determine a cause-and-effect relationship between CTRP gene expression and inflammatory genes and obesity indices. Regarding a possible gender effect on gene expression patterns in obese patients, more studies with greater sample size should be performed to evaluate the roles of CTRPs in both obese women and obese men. Most of all, the mechanistic studies could help us to understand the detailed role of CTRPs in the pathogenesis of obesity.

In this study, we found that CTRP3 and CTRP9 mRNA expression was greater in obese women than in controls. More importantly, CTRP3 and CTRP9 expression significantly correlated with inflammatory parameters and obesity indices. Hence, it seems that CTRP3 and CTRP9 have important roles in glucose metabolism and obesity-related conditions such as inflammation. However, more clinical studies are required to clarify the relationships of CTRP3 and CTRP9 with glucose, metabolic disorders, and obesity-related inflammation.

\section{Acknowledgment}

We thank the supervisors of the advanced laparoscopic surgeries centers at Loqman Hakim and Sina hospitals of Tehran University of Medical Sciences for their helpful cooperation. We are grateful to all of our patients who participated in this study.

6. Seldin MM, Tan SY, Wong GW. Metabolic function of the CTRP family of hormones. Reviews in Endocrine and Metabolic Disorders. 2014;15(2):111-23.

7. Schäffler A, Buechler C. CTRP family: linking immunity to metabolism. Trends Endocrinol Metab. 2012;23(4):194-204.

8. Li Y, Wright GL, Peterson JM. C1q/TNF-related protein 3 (CTRP3) function and regulation. Compr Physiol. 2017;7(3):863-878.

9. Wei Z, Lei X, Petersen PS, Aja S, Wong GW. Targeted deletion of C1q/TNF-related protein 9 (CTRP9) increases food intake, decreases insulin sensitivity, and promotes hepatic steatosis in mice. Am J Physiol Endocrinol Metab. 2014;306(7):e779-90. 
10. Peterson JM, Wei Z, Wong GW. Clq/TNFrelated protein-3 (CTRP3), a novel adipokine that regulates hepatic glucose output. J Biol Chem. 2010;285(51):39691-701.

11. Li X, Jiang L, Yang M, Wu Y-w, Sun S-x, Sun J-z. CTRP3 modulates the expression and secretion of adipokines in 3T3-L1 adipocytes. Endocr J. 2014;61(12):1153-62.

12. Akiyama H, Furukawa S, Wakisaka S, Maeda T. CTRP3/cartducin promotes proliferation and migration of endothelial cells. Mol Cell Biochem. 2007;304(1-2):243-8.

13. Hou Q, Lin J, Huang W, Li M, Feng J, Mao X. CTRP3 Stimulates Proliferation and Anti-Apoptosis of Prostate Cells through PKC Signaling Pathways. PloS one. 2015;10(7):e0134006.

14. Wolf RM, Lei X, Yang Z-C, Nyandjo M, Tan SY, Wong GW. CTRP3 deficiency reduces liver size and alters IL-6 and TGF $\beta$ levels in obese mice. Am J Physiol Endocrinol Metab. 2016;310(5):E332-45.

15. Li X, Jiang L, Yang M, Wu Y-w, Sun S-X, Sun J-z. Expression of CTRP3, a novel adipokine, in rats at different pathogenic stages of type 2 diabetes mellitus and the impacts of GLP-1 receptor agonist on it. Journal of diabetes research. 2014;2014(9933):398518.

16. Wong GW, Krawczyk SA, KitidisMitrokostas C, Ge G, Spooner E, Hug C, et al. Identification and characterization of CTRP9, a novel secreted glycoprotein, from adipose tissue that reduces serum glucose in mice and forms heterotrimers with adiponectin. FASEB J. 2009;23(1):241-58.

17. Peterson JM, Wei Z, Seldin MM, Byerly MS, Aja S, Wong GW. CTRP9 transgenic mice are protected from diet-induced obesity and metabolic dysfunction. Am J Physiol Regul Integr Comp Physiol. 2013;305(5);R522-33.

18. Moradi N, Fadaei R, Emamgholipour S, Kazemian E, Panahi G, Vahedi S, et al. Association of circulating CTRP9 with soluble adhesion molecules and inflammatory markers in patients with type 2 diabetes mellitus and coronary artery disease. PloS one. 2018;13(1):e0192159.

19. Thomas D Schmittgen, Livak KJ. Analyzing real-time PCR data by the comparative CT method. Nature Protocols. 2008;3:1101-8.
20. Wagner RM, Sivagnanam K, Clark WA, Peterson JM. Divergent relationship of circulating CTRP3 levels between obesity and gender: a crosssectional study. PeerJ. 2016;4:e2573.

21. Ban B, Bai B, Zhang M, Hu J, Ramanjaneya M, Tan BK, et al. Low serum cartonectin/CTRP3 concentrations in newly diagnosed type 2 diabetes mellitus: in vivo regulation of cartonectin by glucose. PloS one. 2014;9(11):e112931.

22. Li X, Jiang L, Yang M, Wu Yw, Sun Jz, Sun Sx. CTRP3 improves the insulin sensitivity of 3T3-L1 adipocytes by inhibiting inflammation and ameliorating insulin signalling transduction. Endokrynol Pol. 2014;65(4):252-8.

23. Nishimoto $H$, Yamamoto A, Furukawa $S$, Wakisaka S, Maeda T. C1q/TNF-related protein 3 expression and effects on adipocyte differentiation of 3T3-L1 cells. Cell Biol Int. 2017;41(2):197-203.

24. Schmid A, Kopp A, Hanses F, Bala M, Müller $\mathrm{M}$, Schäffler A. The novel adipokine C1q/TNFrelated protein-3 is expressed in human adipocytes and regulated by metabolic and infection-related parameters. Exp Clin Endocrinol Diabetes. 2012;120(10):611-7.

25. Kwon MR, Cress E, Clark WA, Alamian A, Lu Y, Peterson JM. The adipokine Clq TNF related protein 3 (CTRP3) is elevated in the breast milk of obese mothers. PeerJ. 2018;6:e4472.

26. Qu H, Deng M, Wang H, Wei H, Liu F, Wu J, et al. Plasma CTRP-3 concentrations in Chinese patients with obesity and type II diabetes negatively correlate with insulin resistance. J Clin Lipidol. 2015;9(3):289-94.

27. Forouhi N, Saedisomeolia A, Djalali M, Eshraghian MR, Morshedzadeh N, Zabetian-Targhi $\mathrm{F}$, et al. Serum $\mathrm{Clq}$ and tumor necrosis factor (TNF)-related protein 9 in women with Polycystic Ovary Syndrome. Diabetes Metab Syndr. 2016;10(2):S131-4.

28. Kanasaki K, Koya D. Biology of obesity: lessons from animal models of obesity. J Biomed Biotechnol. 2011;2011:197636.

29. Deng W, Li C, Zhang Y, Zhao J, Yang M, Tian $M$, et al. Serum Clq/TNF-related protein-3 (CTRP3) levels are decreased in obesity and hypertension and are negatively correlated with parameters of insulin resistance. Diabetol Metab Syndr. 2015;7:33. 
30. Wolf RM, Steele KE, Peterson LA, Magnuson TH, Schweitzer MA, Wong GW. Lower circulating C1q/TNF-related protein-3 (CTRP3) levels are associated with obesity: a cross-sectional study. PLoS One. 2015;10(7):e0133955.

31. Zhang J, Zhang B, Cheng Y, Xu J. Low serum CTRP3 levels are associated with nonalcoholic fatty liver disease in patients with type 2 diabetes mellitus. Cytokine. 2018;106:131-135.

32. Curat CA, Wegner V, Sengenès $C$, Miranville A, Tonus C, Busse R, et al. Macrophages in human visceral adipose tissue: increased accumulation in obesity and a source of resistin and visfatin. Diabetologia. 2006;49(4):744-7.

33. Lin J, Liu Q, Zhang H, Huang X, Zhang R, Chen $\mathrm{S}$, et al. Clq/Tumor necrosis factor-related protein-3 protects macrophages against LPS-induced lipid accumulation, inflammation and phenotype transition via PPAR $\gamma$ and TLR4-mediated pathways. Oncotarget. 2017;8(47):82541-82557.

34. Weigert J, Neumeier M, Schaffler A, Fleck M, Scholmerich J, Schutz C, et al. The adiponectin paralog CORS-26 has anti-inflammatory properties and is produced by human monocytic cells. FEBS Lett. 2005;579(25):5565-70.

35. Trayhurn P, Wood IS. Adipokines: inflammation and the pleiotropic role of white adipose tissue. Br J Nutr. 2004;92(3):347-55.

36. Hwang YC, Oh SW, Park SW, Park CY. Association of serum C1q/TNF-Related Protein-9 (CTRP9) concentration with visceral adiposity and metabolic syndrome in humans. Int $\mathrm{J}$ Obes. 2014;38(9):1207-12.

37. Wang J, Hang T, Cheng X-m, Li D-m, Zhang Q-g, Wang L-j, et al. Associations of Clq/TNF- related protein-9 levels in serum and epicardial adipose tissue with coronary atherosclerosis in humans. Biomed Res Int. 2015;2015:971683.

38. Wolf RM, Steele KE, Peterson LA, Zeng X, Jaffe AE, Schweitzer MA, et al. C1q/TNF-related protein-9 (CTRP9) levels are associated with obesity and decrease following weight loss surgery. J Clin Endocrinol Metab. 2016;101(5):2211-7.

39. Li YX, Run L, Shi T, Zhang YJ. CTRP9 regulates hypoxia-mediated human pulmonary artery smooth muscle cell proliferation, apoptosis and migration via TGF- $\beta 1 / E R K 1 / 2$ signaling pathway. Biochem Biophys Res Commun. 2017;490(4):1319-1325.

40. Moradi N, Fadaei R, Emamgholipour S, Kazemian E, Panahi G, Vahedi S, et al. Association of circulating CTRP9 with soluble adhesion molecules and inflammatory markers in patients with type 2 diabetes mellitus and coronary artery disease. PloS one. 2018;13(1):e0192159.

41. Li J, Zhang P, Li T, Liu Y, Zhu Q, Chen T, et al. CTRP9 enhances carotid plaque stability by reducing pro-inflammatory cytokines in macrophages. Biochem Biophys Res Commun. 2015;458(4):890-5.

42. Zhang P, Huang C, Li J, Li T, Guo H, Liu T, et al. Globular CTRP9 inhibits oxLDL-induced inflammatory response in RAW 264.7 macrophages via AMPK activation. Mol Cell Biochem. 2016;417(1-2):67-74.

43. Christiansen T, Richelsen B, Bruun JM. Monocyte chemoattractant protein-1 is produced in isolated adipocytes, associated with adiposity and reduced after weight loss in morbid obese subjects. Int J Obes. 2005;29(1):146-50. 